\title{
Metabolites from Resistant and Susceptible Pinus thunbergii after Inoculation with Pine Wood Nematode
}

\author{
Fujia Zhang ${ }^{1}$, Junko Kajiwara ${ }^{1}$, Yasuhiro Mori ${ }^{2}$, Mineko Ohira ${ }^{3}$, Yuji Tsutsumi ${ }^{*}$, Ryuichiro Kondo ${ }^{1}$ \\ ${ }^{1}$ Department of Forest and Forest Products Sciences, Faculty of Agriculture, Kyushu University, Fukuoka, Japan; ${ }^{2}$ Fukuoka Prefec- \\ ture Forest Research and Extension Center, Kurume, Japan; ${ }^{3}$ Forest Tree Breeding Center, Forestry and Forest Products Research \\ Institute, Ibaraki, Japan. \\ Email: *y-tsutsu@agr.kyushu-u.ac.jp
}

Received January $5^{\text {th }}, 2013$; revised February $7^{\text {th }}, 2013$; accepted February $14^{\text {th }}, 2013$

\begin{abstract}
Pine wilt disease (PWD), which is caused by pine wood nematodes (PWN), is one of the most serious forest diseases worldwide. To clarify the mechanism of resistance to PWD, we compared metabolites from resistant and susceptible Japanese black pine (Pinus thunbergii) families after inoculation with PWN. After 2 weeks to 1 month post inoculation, the number of PWN dramatically increased in susceptible plants, but not in resistant plants. At this PWN-proliferation phase, ethyl acetate soluble fractions extracted from PWN-inoculated plants were analyzed by gas chromatogramphymass spectrometry (GC-MS). Although most compounds were qualitatively and quantitatively similar between resistant and susceptible plants, resistant plants accumulated 2.0-fold more linoleic acid (LA) than susceptible plants. On the other hand, benzoic acid (BA) was barely detected in resistant plants, but it accumulated in susceptible plants as the number of PWN increased. Susceptible plants contained greater levels of the nematicidal compounds pinosylvin and pinosylvin monomethyl ether, compared with resistant plants. These results suggested that LA is involved in the resistance reaction against PWN-proliferation, and that BA could be a good biomarker for PWD.
\end{abstract}

Keywords: Benzoic Acid; Bursaphelenchus xylophilus; Linoleic Acid; Pinosylvin; Pinus thunbergii; Resistance

\section{Introduction}

Pine wood nematode (PWN) (Bursaphelenchus xylophilus) is the causal pathogen of pine wilt disease (PWD) [1], the most serious disease in East Asian pine forests [2-4]. This disease has been also found in USA $[5,6]$, Canada [7], Nigeria [8], Mexico [9], Portugal [10] and Spain [11]. Therefore, it is a threat to pine forests worldwide.

A breeding program to produce Japanese black pine (Pinus thunbergii) and Japanese red pine (P. densiflora) resistant to PWN began in 1978 [12]. So far, seedlings have been produced commercially from a seed orchard of resistant clones. In inoculation trials, these seedlings were relatively resistant; $47 \%-62 \%$ survived after inoculation with PWN, compared with $22 \%$ - $55 \%$ of nonorchard seedlings $[13,14]$. Some orchard seedlings were highly resistant, and plants that were clonally propagated from them were barely damaged by a virulent PWN isolate $[15,16]$. However, it is difficult to predict whether these plants will retain resistance against as-yet undiscovered isolates or extremely virulent isolates [17] for

${ }^{*}$ Corresponding author. many decades. As long as the mechanism of resistance is unknown, the breeding program must continue to select resistant plants via inoculation with the most virulent isolate known at the time. To save time and expense in the program, it is important to identify the factors involved in PWD resistance.

Numerous PWN were detected in susceptible pine trees after inoculation, whereas far fewer PWN were locally detected in resistant trees [18-20]. These observations could be explained by the hypothesis that narrow resin canals of resistant pine trees function as bottlenecks for PWN-migration. This hypothesis was based on crosssectional observations of the main migration pathways, i.e., cortical resin canals [21]. However, our previous study demonstrated that symptom development in pine trees was unaffected by mimicking inhibited or free PWN migration [22]. This suggested that resistance was independent of PWN migration. The PWN population drastically increased in susceptible pine stem cuttings by 20 days after inoculation (dai), while it remained unchanged or gradually decreased in resistant cuttings [22]. Thus, it is likely that resistance depends on factors that inhibit the proliferation of $\mathrm{PWN}$, rather than migration. 
Although some secondary metabolites have been considered as nematicidal factors, little is known about the metabolites involved in resistance mechanisms. Stilbenoids (e.g., pinosylvin monomethyl ether and 3-O-methyldihydropinosylvin) were specifically detected in resistant pine species (e.g., P. massoniana, P. strobus, and P. palustris) in previous studies $[23,24]$. These compounds showed acute nematicidal activity against PWN. However, it has not been proven that they inhibit PWN proliferation, or they are specific in resistant pines. It is possible that unknown compounds from resistant pine trees inhibit PWN proliferation by disrupting their life cycle and/or reproduction.

Here, we compared the metabolites from resistant and susceptible PWN-inoculated P. thunbergii plants using GC-MS, and examined the relationship between resistant- and susceptible-specific compounds and PWN proliferation.

\section{Materials and Methods}

\subsection{Plant Materials}

Two-year old resistant and susceptible $P$. thunbergii plants were grown in the nurseries of the Forest Tree Breeding Center (Kumamoto prefecture). Open-pollinated seedlings from "Namikata-37" and "Misaki-90" families were used as resistant plants, based on previous reports $[14,25,26]$. Open-pollinated seedlings from " $\mathrm{Ku}$ nisaki-131" and "Kimotsuki-24" families were used as susceptible plants because the proportion of damaged seedlings was $88.1 \%(74 / 84)$ and $83.6 \%(117 / 140)$, respectively, after the inoculations described below.

\subsection{Inoculation with Pine Wood Nematodes}

A PWN isolate, "Shimabara" [12], was cultured on Botrytis cinerea grown on autoclaved barley grains media in Petri dishes for $7-10$ days at $25^{\circ} \mathrm{C}$ in the dark. The PWN were isolated by the Baermann funnel technique [27] and the PWN-suspension was adjusted to 100,000 PWN per ml with distilled water. Then, $50 \mu \mathrm{l}$ of the suspension (i.e., $5000 \mathrm{PWN}$ ) was inoculated into a peeled wound on the main stem at about $5 \mathrm{~cm}$ high from ground. Inoculations were conducted in late July for two consecutive years.

\subsection{Counting the Pine Wood Nematodes}

In the first year, PWN were isolated from four segments $(0-1,5-6,10-11$, and $15-16 \mathrm{~cm}$ away from the inoculation point) of each plant at 7,15 , and 29 dai by the Baermann funnel technique [27]. In the second year, PWN were isolated from the same points as those in the first year at 7, 17, and 31 dai. The isolated PWN were counted under a stereomicroscope at $40 \times$ magnification.

\subsection{Extraction of Metabolites from Inoculated Plants}

To identify the compounds associated with PWN proliferation, we analyzed metabolites from the segments adjacent to those sampled to monitor the number of PWN. Two segments ( $1-2$ and $6-8 \mathrm{~cm}$ away from the inoculation point) in the first year and three segments $(1-3,6-8$, and $11-13 \mathrm{~cm}$ away from the inoculation point) in the second year were harvested for further experiments from the samples after counting PWN. All segments were stripped, and bark and xylem were separately ground in liquid nitrogen. Homogenates were extracted in triplicate with $30 \mathrm{ml}$ methanol for $3 \mathrm{~h}$ at room temperature. The solvent was removed from the filtrate extracts under reduced pressure using a rotary evaporator. Methanol extracts were further concentrated under a stream of nitrogen gas. Subsequently, extracts were dissolved in $15 \mathrm{ml}$ ethyl acetate. Soluble fractions were concentrated by removing the solvent using the above-mentioned method.

\subsection{Gas Chromatography Mass Spectrometry Analysis}

We added $30 \mu \mathrm{g} n$-docosane as an internal standard to 1 $\mathrm{mg}$ of the ethyl acetate soublel fractions. They were trimethylsilylated with $\mathrm{N}, \mathrm{O}$-bis-(trimethylsilyl)-trifluoroacetamide in pyridine for $10 \mathrm{~min}$ at $70^{\circ} \mathrm{C}$. Gas chromatography-mass spectrometry (GC-MS) was performed on a GC 17-A/QP5050 (Shimadzu, Kyoto, Japan) equipped with an autoinjector and a column (SPB-5 L $\times$ I.D. $30 \mathrm{~m} \times$ $0.20 \mathrm{~mm}, \mathrm{~d}_{\mathrm{f}} 0.20 \mu \mathrm{m}$, Sigma-Aldrich Co. LLC). We injected $1 \mu \mathrm{l}$ of the derivatized sample into the column. The temperature program was as follows: initial temperature of $120^{\circ} \mathrm{C}$, held for $3 \mathrm{~min}$, increased at $10^{\circ} \mathrm{C} / \mathrm{min}$ to $320^{\circ} \mathrm{C}$, and held for $3 \mathrm{~min}$. The carrier gas was helium. Compounds were identified as trimethylsilylated derivatives by comparing their mass spectra with those in the GC-MS spectral library (NIST62). We used a linear calibration curve to convert the peak area ratio (compound/internal standard) for each identified compound into the concentration of that compound in $1 \mathrm{mg}$ ethyl acetate-soluble fraction. We also calculated the concentration of each compound in $1 \mathrm{~g}$ extractive-free wood meal.

\section{Results}

\subsection{PWN-Proliferation in Resistant and Susceptible Plants}

We compared the number of PWN between resistant and susceptible seedlings after inoculation. In the first and the second years, there was no significant increase in the number of PWN in both resistant and susceptible seed- 
lings at 7 dai (Figure 1). However, dramatic increases of PWN-population were observed in two susceptible families after 15 and 31 dai in the first and the second year, respectively. In susceptible seedlings after PWN-inoculation conducted in the first (a) and the second year (b). LA concentration was determined in xylem extracts of two segments (1-2 and $6-8 \mathrm{~cm}$ away from inoculation point) in the first year and three segments $(1-3,6-8$, and $11-13 \mathrm{~cm}$ away from inoculation point) in the second year. Values are means of the first year; the rate of increase of the PWN population was greater than that in the second year. Within the experimental period of 30 days each year, there was no increase in the PWN-population in two resistant families.

\subsection{Quantitative Determination of Resistant and Susceptible-Specific Compounds}

We preliminarily conducted GC-MS analyses of ethyl

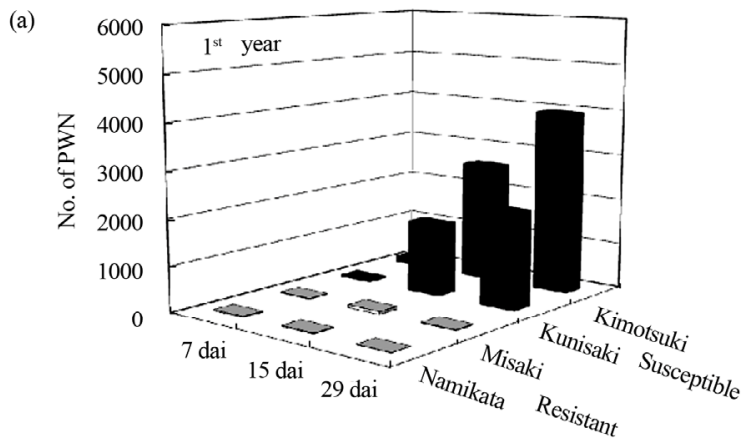

(b)

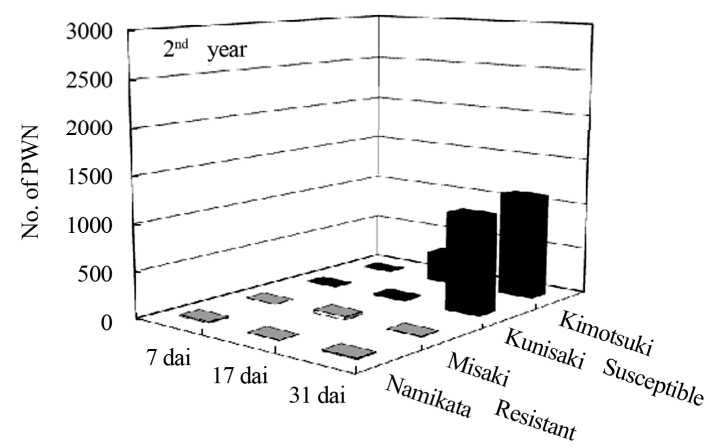

Figure 1. Numbers of PWN detected from resistant and susceptible seedlings after PWN-inoculation in the first (a) and the second year (b). PWN were detected from four $1 \mathrm{~cm}$ long segments $(0-1 \mathrm{~cm}, 5-6 \mathrm{~cm}, 10-11 \mathrm{~cm}$, and $15-16 \mathrm{~cm}$ away from inoculation point) at three different time points in both years. Values are mean numbers (per segment) of three seedlings from two resistant families ("Namikata-37" and "Misaki-90", grey columns) or two susceptible families ("Kunisaki-131" and "Kimotsuki-24", black columns). At 15 and 29 dai in the first year and 31 dai in the second year, there were significant differences in the number of PWN between resistant and susceptible families by t-test ( $p<$ 0.05). dai, Days after inoculation. acetate extracts from two resistant plants and a susceptible plant. There were few differences in the bark metabolites among resistant and susceptible plants. However, the xylem metabolites differed between resistant and susceptible plants. Comparison with spectra in the MS library showed that linoleic acid (LA) trimethylsilyl ester was commonly detected in xylem extracts of two resistant plants. It was also detected in the methylated samples instead of trimethylsilylated ones. In this preliminary analysis, LA was the metabolite that was detected specifically and commonly in resistant plants.

Based on these findings, we quantified LA in the xylem of seedlings from two resistant and two susceptible families (Figure 2). In both the first and the second years, the LA concentrations were similar within a family, but differed between resistant and susceptible families. The resistant seedlings accumulated 2.7- and 2.0-fold more LA than did susceptible seedlings in the first and the second year, respectively. In our preliminarily GC-MS analyses, we also found that benzoic acid (BA) was the typical metabolite in susceptible families, instead of LA in resistant one. Kawazu et al. [28] reported that this compound accumulated to high levels in $P$. thunbergii trees after PWN-inoculation, thus we conducted determination of BA. In the first year, BA was barely detected
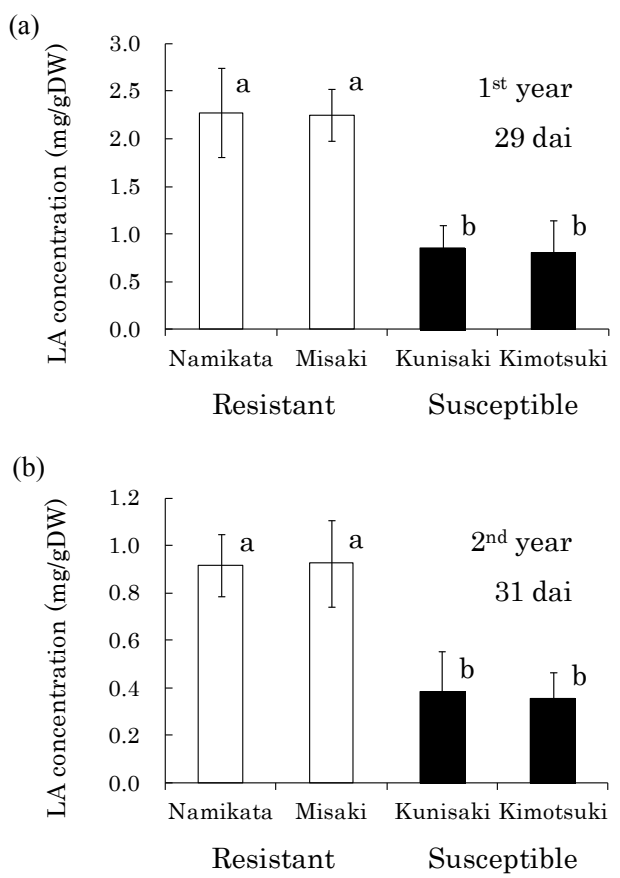

Figure 2. Linoleic acid (LA) concentrations in resistant and LA concentrations in all segments of individual seedling. Error bars indicate SE (standard error) of four seedlings in the first year and three in the second year. White and black bars show 'resistant' and 'susceptible' families, respectively. Significant differences are indicated by different letters (a, b) using a LSD multiple comparison ( $<<0.05)$. dai, Days after inoculation. 
in xylem extracts from the resistant family "Namikata37" (Figure 3(a)). The other resistant family "Misaki90" accumulated no BA. On the other hand, we detected higher concentrations of BA in susceptible seedlings. However, one seedling from a susceptible family "Kunisaki131", which did not allow PWN to proliferate, exhibited a very low BA level. In the second year, 8 out of 10 susceptible seedlings produced high levels of BA, while resistant seedlings barely produced any BA (Figure 3(b)). BA was hardly detected in the susceptible seedlings in which few PWN proliferated. There were significant positive correlations between the number of PWN and BA concentration in each seedling (correlation coefficients were $0.90(\mathrm{p}<0.01)$ and $0.54(\mathrm{p}<0.05)$ in the first and the second year, respectively).

We also determined the concentrations of pinosylvin and pinosylvin monomethyl ether in resistant and susceptible seedlings, because these compounds were reported to have nematicidal activities in previous studies

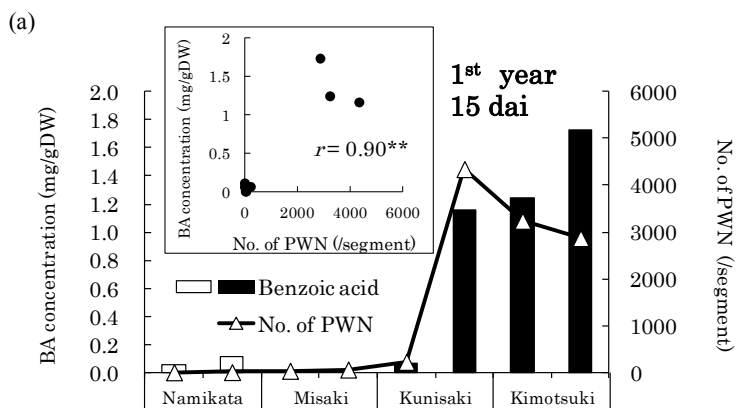

$$
\text { Resistant Susceptible }
$$

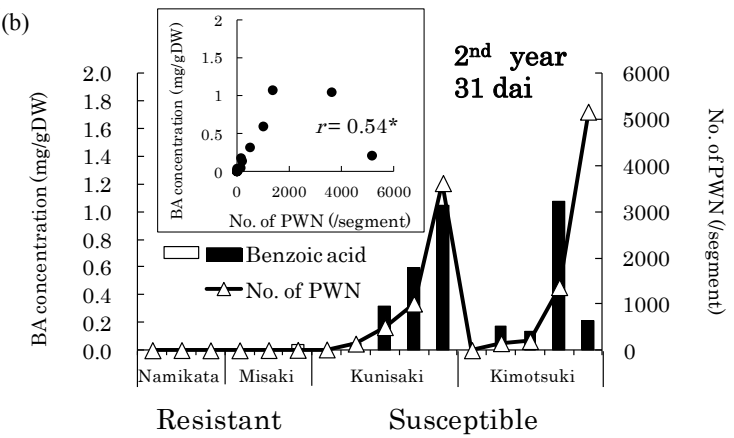

Figure 3. Relationships between benzoic acid (BA) concentrations and numbers of PWN after PWN-inoculation in the first (a) and the second year (b). BA concentration was determined in whole extracts (mixture of bark and xylem) of two segments $(1-2$ and $6-8 \mathrm{~cm}$ away from inoculation point) in the first year and three segments $(1-3,6$ - 8 and $11-13 \mathrm{~cm}$ away from inoculation point) in the second year. Values are BA concentrations in all segments of individual seedlings. White and black bars show "resistant" and "susceptible" seedlings, respectively. PWN were counted in four $1 \mathrm{~cm}$ long segments $(0-1 \mathrm{~cm}, 5-6 \mathrm{~cm}, 10-11 \mathrm{~cm}$, and 15 $16 \mathrm{~cm}$ away from inoculation point) in both years. Values are total number of PWN in individual seedlings. dai, Days after inoculation.
$[23,29]$. There were very high levels of these compounds in susceptible seedlings at both 15 and 29 dai (Figure 4). In particular, pinosylvin accumulated to very high levels in one of the susceptible families, "Kunisaki-131", during PWN-proliferation (15 - 29 dai).

\section{Discussion}

In previous studies, the PWN population dramatically increased at 2 - 4 weeks after inoculation in non-resistant $P$. thunbergii seedlings [30], and visible symptoms of PWD were observed after 19 dai [31]. Similar to those reports, we observed marked increases in the abundance of PWN in susceptible plants after 15 and 31 dai in the first and the second year, respectively (Figure 1). Thus,

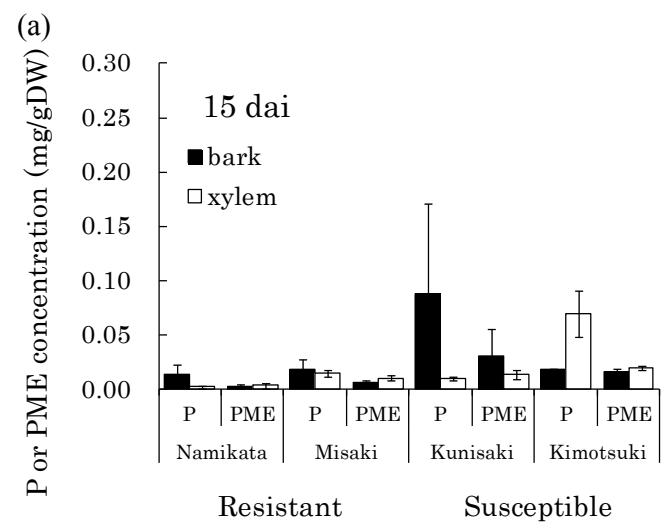

(b)

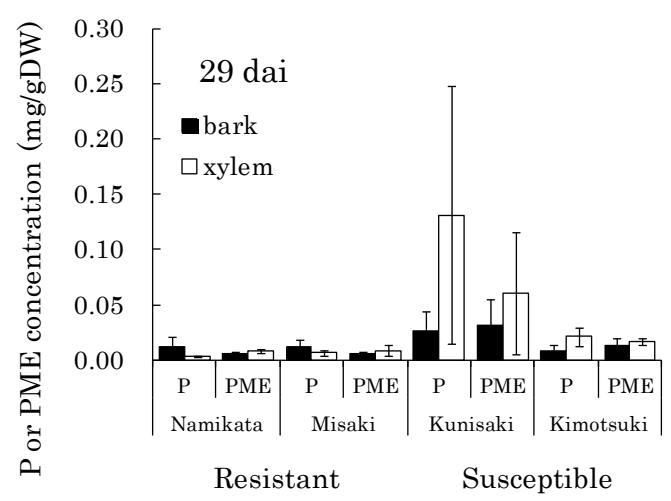

Figure 4. Pinosylvin (P) and pinosylvin monomethyl ether (PME) concentrations in resistant and susceptible seedlings at 15 (a) and 29 (b) days after PWN-inoculation in the first year. Concentrations of these compounds were separately determined in bark and xylem extracts from a segment (6 $8 \mathrm{~cm}$ away from inoculation point). Valuesare means of $P$ or PME concentrations in the segment of individual seedlings. Error bars indicate SE (standard error) of two and four seedlings at 15 and 29 dai, respectively. In each bar, black part shows concentration in bark and white part shows concentration in xylem. At 15 dai, there were significant differences in PME concentration between resistant and susceptible families by t-test $(p<0.05)$. dai, Days after inoculation. 
we focused on the metabolites in pine trees during the PWN-proliferation phase (15 - 31 dai).

In the preliminary GC-MS analysis of metabolites, LA was the only compound that was specifically and commonly detected in resistant xylem but not in susceptible xylem. Quantitative analysis of LA revealed that there were no differences in LA concentration between two resistant families, and resistant families produced 2.7fold more LA than susceptible families at 29 dai in the first year (Figure 2(a)). This significant gap between resistant and susceptible families was reproduced at 31 dai in the next year (Figure 2(b)), even though different individuals from each family were analyzed. These results suggested that LA is a key compound associated with the resistance reaction against PWN during the phase when resistant plants inhibit PWN-proliferation. However, authentic LA did not show nematicidal activeties, nor did it inhibit PWN-reproduction in vitro (unpublished data). In pepper (Capsicum annuum), 9-lipoxygenases were crucial for defense responses against infection of a pathogen (Xanthomonas campestris pv. Vesicatoria), and their activity was associated with LA peroxidation [32]. Recently, Nose and Shiraishi [33] compared four SAGE (serial analysis of gene expression) P. thunbergii libraries; from PWN-inoculated resistant plants, non-inoculated resistant plants, inoculated non-resistant plants, and non-inoculated non-resistant plants. This transcriptome analysis showed that lipoxygenase-5 was specifically up-regulated in PWN-inoculated resistant pine. In an in vitro assay using callus tissues of pine trees, malondialdehyde (an indicator of lipoperoxidation) accumulated to high levels in PWN-inoculated callus from the resistant pine species $P$. taeda, compared with its accumulation in non-inoculated callus [34]. Thus, LA itself may not directly inhibit PWN-proliferation, however, its metabolites though lipoperoxidation may be related to the $\mathrm{PWN}$ resistance mechanism in pine trees.

We also detected high levels of BA in susceptible seedlings, while it was barely detectable in resistant seedlings (Figure 3). Significant correlations between the number of PWN and BA concentration were seen in both the first and the second year. These results suggest that BA accumulation is associated with the increase in abundance of PWN. Ueda et al. [35] first reported the accumulation of BA in naturally $\mathrm{PWN}$-infected $P$. densiflora leaves and branches. According to Kawazu [28], when 3-year-old $P$. thunbergii seedlings were inoculated with PWN, BA gradually accumulated as the PWN-population increased. Our observations were consistent with these reports. Taken together, these findings strongly suggest that BA is a good biomarker for PWD. One possible explanation for this observation is that resistant plants might metabolize BA into an unidentified antinematode compound. The fate of $\mathrm{BA}$ in resistant pines is of interest for understanding the expression of resistant mechanisms.

Stilbenoids are thought to play roles in the resistance reaction, because they show nematicidal activities against PWN $[23,24,29]$. In fact, pinosylvin monomethyl ether and 3-O-methyldihydropinosylvin were detected in methanol extracts from resistant pine species ( $P$. massoniana, P. strobus, and P. palustris) $[23,24]$. However, to date, the stilbenoid concentrations have not been quantitatively compared between resistant and susceptible pine trees. In this study, we detected higher levels of pinosylvin and pinosylvin monomethyl ether in susceptible seedlings than in resistant seedlings during $15-29$ dai (Figure 4). If stilbenoids inhibit PWN-proliferation, then this observation cannot explain the dramatic increase in abundance of PWN in susceptible seedlings compared to resistant seedlings. Therefore, acute nematicidal compounds observed pervious in vitro assay do not necessarily play a major role in resistance mechanisms, including inhibition of PWN-proliferation.

\section{Conclusion}

LA was suggested as a candidate compound for resistance to PWD, because it accumulated in resistant plants, but not susceptible plants. Although LA does not have acute nematicidal activities in in vitro assays, it leads to generation of reactive oxygen species though lipoperoxidation, which may participate in the resistance reaction in vivo. Combining previous analyses $[32,36]$ with our more recent analyses may help to further our understanding of the resistance mechanism against PWD.

\section{REFERENCES}

[1] T. Kiyohara and Y. Tokushige, "Inoculation Experiments of a Nematode, Bursaphelenchus spp., onto Pine Trees," Journal of the Japanese Forest Society, Vol. 53, No. 7, 1971, pp. 210-218.

[2] B. Yang and Q. Wang, "Distribution of the Pinewood Nematode in China and Susceptibility of Some Chinese and Exotic Pines to the Nematode," Canadian Journal of Forest Research, Vol. 19, No. 12, 1989, pp. 1527-1530. doi:10.1139/X89-232

[3] C. K. Yi, B. H. Byun, J. D. Park, S. I. Yang and K. H. Chang, "First Finding of the Pine Wood Nematode, Bursaphelenchus Xylophilus (Steiner et Buhrer) Nickle and Its Insect Vector in Korea," The Research Reports of the Forestry Research Institute, Vol. 38, 1989, pp. 141-149.

[4] Y. Kishi, "The Pine Wood Nematode and the Japanese Pine Sawyer," Thomas Company, Tokyo, 1995, p. 302.

[5] J. C. Adams and A. L. Morehart, "Decline and Death of Pinus spp. in Delaware Caused by Bursaphelenchus xylophilus," Journal of Nematology, Vol. 14, No. 3, 1982, pp. 383-385.

[6] L. D. Dwinell, "Relative Susceptibilities of Five Pine 
Species to Three Populations of the Pinewood Nematode," Plant Disease, Vol. 69, No. 5, 1985, pp. 440-442.

[7] K. Knowles, Y. Beaubien, M. J. Wingfield, F. A. Baker and D. W. French, "The Pinewood Nematode New in Canada," The Forestry Chronicle, Vol. 59, 1983, p. 40.

[8] F. A. Khan, "The Pine Wilt Disease Caused by Bursaphelenchus xylophilus in Nigeria," Pakistan Journal of Nematology, Vol. 9, 1991, pp. 57-59.

[9] L. D. Dwinell, "First Report of Pinewood Nematode (Bursaphelenchus xylophilus) in Mexico," Plant Disease, Vol. 77, No. 8, 1993, p. 846. doi:10.1094/PD-77-0846A

[10] M. M. Mota, H. Braasch, M. A. Bravo, A. C. Penas, W. Burgermeister, K. Metge and E. Sousa, "First Report of Bursaphelenchus xylophilus in Portugal and in Europe," Nematology, Vol. 1, No. 7, 1999, pp. 727-734. doi: $10.1163 / 156854199508757$

[11] L. Robertson, S. C. Arcos, M. Escuer, R. S. Merino, G. Esparrago, A. Abellera and A. Navas, "Incidence of the Pinewood Nematode Bursaphelenchus xylophilus Steiner \& Buhrer, 1934 (Nickle, 1970) in Spain," Nematology, Vol. 13, No. 6, 2011, pp. 755-757.

[12] Y. Fujimoto, T. Toda, K. Nishimura, H. Yamate and S. Fuyuno, "Breeding Project on Resistance to Pine-Wood Nematode-An Outline of the Research and the Achievement of Project for Ten Years," Bulletin of the Forest Tree Breeding Institute, Vol. 7, 1989, pp. 1-84.

[13] T. Toda and S. Kurinobu, "Realized Genetic Gains Observed in Progeny Tolerance of Selected Red Pine (Pinus densiflora) and Black Pine (P. thunbergii) to Pine Wilt Disease," Silvae Genetica, Vol. 51, No. 1, 2002, pp. 4244.

[14] S. Goto, F. Miyahara and Y. Ide, "Contribution of Pollen Parents to the Nematode-Resistance in Seedlings of Japanese Black Pine," Journal of the Japanese Forest Society, Vol. 84, No. 1, 2002, pp. 45-49.

[15] Y. Mori, F. Miyahara and S. Goto, "Improving the Pine Wilt Disease Resistance of Cutting-Propagated Japanese Black Pine Plantlet Populations by Inoculating Ortets with Pinewood Nematodes," Journal of the Japanese Forest Society, Vol. 88, No. 3, 2006, pp. 197-201. doi:10.4005/jjfs.88.197

[16] Y. Mori, F. Miyahara and S. Goto, "The Effect of Rooted Cutting Propagation of Non-Damaged Japanese Black Pine through the Inoculation Test with the Pinewood Nematode on Nematode-Resistant Plant Production," Proceeding of the International Symposium "Pine Wilt Disease: A Worldwide Threat to Forest Ecosystems", Lisbon, 10-14 July 2006, pp. 52-53.

[17] M. Ohira, F. Miyahara, Y. Mori, M. Okawa, J. Miyazaki, S. Masaki, K. Yoshimoto, Y. Sasaki, Y. Yamada, Y. Mitsugi, T. Tagami, T. Koyama, M. Miyazato, M. Tobase, K. Kuroda, M. Okamura, K. Matsunaga and S. Shiraishi, "Development of a Novel Cutting-Production System for Second-Generation Pinus thunbergii Resistant to Pine Wilt Disease," Forest Tree Breeding, Vol. 235, 2010, pp. $1-5$.

[18] P. J. Bedker, M. J. Wingfield and R. A. Blanchette, "Pathogenicity of Bursaphelenchus xylophilus on Three Species of Pine," Canadian Journal of Forest Research, Vol. 17,
No. 1, 1987, pp. 51-57. doi:10.1139/x87-010

[19] H. Oku, T. Shiraishi and K. Chikamatsu, "Active Defense as a Mechanism of Resistance in Pine Against Pine Wilt Disease," Annals of the Phytopathological Society of Japan, Vol. 55, No. 5, 1989, pp. 603-608. doi:10.3186/jijphytopath.55.603

[20] K. Kuroda, "Inhibiting Factors of Symptom Development in Several Japanese Red Pine (Pinus densiflora) Families Selected as Resistant to Pine Wilt," Journal of Forest Research, Vol. 9, No. 3, 2004, pp. 217-224. doi:10.1007/s10310-004-0076-0

[21] E. Kawaguchi, "Relationship between the Anatomical Characteristics of Cortical Resin Canals and Migration of Bursaphelenchus xylophilus in Stem Cuttings of Pinus thunbergii Seedlings," Journal of the Japanese Forest Society, Vol. 88, No. 4, 2006, pp. 240-244. doi:10.4005/jjfs.88.240

[22] Y. Mori, F. Miyahara, Y. Tsutsumi and R. Kondo, "Relationship between Resistance to Pine Wilt Disease and the Migration or Proliferation of Pine Wood Nematodes," European Journal of Plant Pathology, Vol. 122, No. 4, 2008, pp. 529-538. doi:10.1007/s10658-008-9321-2

[23] T. Suga, S. Ohta, K. Munesada, N. Ide, M. Kurokawa, M. Shimizu and E. Ohta, "Endogenous Pine Wood Nematicidal Substances in Pines, Pinus massoniana, P. strobus and P. palustris," Phytochemistry, Vol. 33, No. 6, 1993, pp. 1395-1401. doi:10.1016/0031-9422(93)85098-C

[24] F. Hanawa, T. Yamada and T. Nakashima, "Phytoalexins from Pinus strobus Bark Infected with Pinewood Nematode, Bursaphelenchus xylophilus," Phytochemistry, Vol. 57, No. 2, 2001, pp. 223-228. doi:10.1016/S0031-9422(00)00514-8

[25] N. Kuramoto, M. Ohira, M. Okamura, Y. Hiraoka, T. Taniguchi, M. Kashiwagi, Y. Inoue and Y. Fujisawa, "Genetic Analysis of Pine Wilt Disease-Resistance Using Interbreeding Families in Japanese Black Pine," 116th the Japanese Forest Society Congress, Hokkaido, 27-30 March 2005, p. 649.

[26] K. Kuroda, M. Ohira, M. Okamura and Y. Fujisawa, "Migration and Population Growth of the Pine Wood Nematode (Bursaphelenchus xylophilus) Related to the Symptom Development in the Seedlings of Japanese Black Pine (Pinus thunbergii) Families Selected as Resistant to Pine Wilt," Journal of the Japanese Forest Society, Vol. 89, No. 4, 2007, pp. 241-248. doi:10.4005/ijfs.89.241

[27] G. Thorne, "Principles of Nematology," McGraw-Hill Book Company Inc., New York, 1961, p. 553.

[28] K. Kawazu, "Change in Constituents of Pine Wood by Infection of Pine Wood Nematode," Nippon Nogeikagaku Kaishi, Vol. 64, No. 7, 1990, pp. 1262-1264. doi:10.1271/nogeikagaku1924.64.1262

[29] T. Kohno, K. Togashi and N. Fukamiya, "The Nematocidal Activity and the Structure-Activity Relationships of Stilbenes," Natural Product Research, Vol. 21, No. 7, 2007, pp. 606-615. doi:10.1080/14786410701369730

[30] K. Kuroda, T. Yamada, K. Mineo and H. Tamura, "Effects of Cavitation on the Development of Pine Wilt Disease Caused by Bursaphelenchus xylophilus," Annals of the Phytopathological Society of Japan, Vol. 54, No. 5, 
1988, pp. 606-615. doi:10.3186/ijphytopath.54.606

[31] N. Kuramoto, M. Sasaki, M. Okamura, Y. Hiraoka, M. Kashiwagi, Y.Inoue and Y. Fujisawa, "Comparison of Symptom Development of Pine Wilt Disease in Half-Sib Progeny of the Tanabe-t54 Open Family Line," Kyushu Journal of Forest Research, Vol. 57, 2004, pp. 228-229.

[32] I. S. Hwang and B. K. Hwang, "The Pepper 9-Lipoxygenase Gene CaLOX1 Functions in Defense and Cell Death Responses to Microbial Pathogens," Plant Physiology, Vol. 152, No. 2, 2010, pp. 948-967. doi:10.1104/pp.109.147827

[33] M. Nose and S. Shiraishi, "Comparison of the Gene Expression Profiles of Resistant and Non-Resistant Japanese Black Pine Inoculated with Pine Wood Nematode Using a Modified Long SAGE Technique," Forest Pathology, Vol. 41, No. 2, 2011, pp. 143-155.

$$
\text { doi:10.1111/j.1439-0329.2010.00646.x }
$$

[34] H. Iwahoria and K. Futai, "Lipid Peroxidation and Ion Exudation of Pine Callus Tissues Inoculated with Pinewood Nematodes," Japanese Journal of Nematology, Vol. 23, No. 2, 1993, pp. 79-89.

[35] T. Ueda, H. Oku, K. Tomita, K. Sato and T. Shiraishi, "Isolation, Identification, and Bioassay of Toxic Compounds from Pine Tree Naturally Infected by Pine Wood Nematode," Annals of the Phytopathological Society of Japan, Vol. 50, No. 2, 1984, pp. 166-175. doi:10.3186/jijphytopath.50.166

[36] H. Shin, H. Lee, K.-S. Woo, E.-W. Noh, Y.-B. Koo and K.-J. Lee, "Identification of Genes Upregulated by Pinewood Nematode Inoculation in Japanese Red Pine," Tree Physiology, Vol. 29, No. 3, 2009, pp. 411-421. doi:10.1093/treephys/tpn034 